\title{
A precise in situ calibration of the RHIC H-Jet polarimeter
}

\section{A.A. Poblaguev}

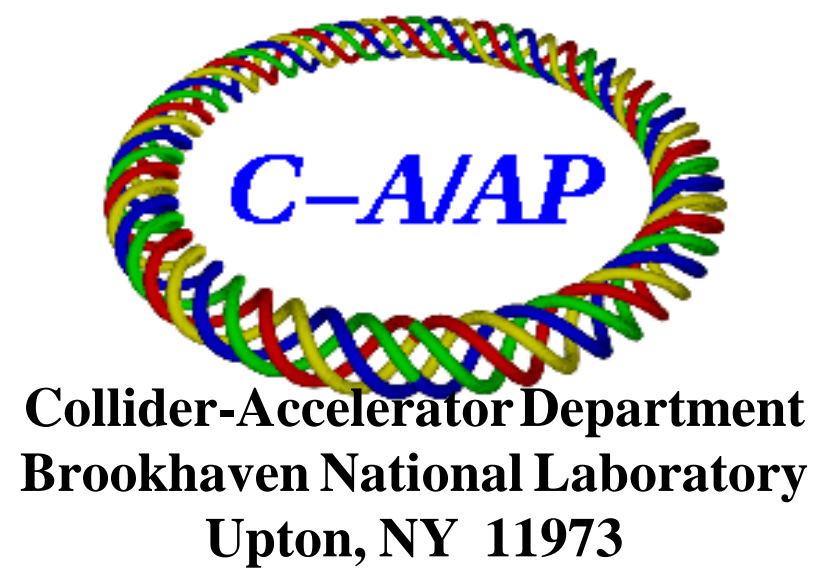

Notice: This document has been authorized by employees of Brookhaven Science Associates, LLC under Contract No. DE-AC02-98CH10886 with the U.S. Department of Energy. The United States Government retains a nonexclusive, paid-up, irrevocable, world-wide license to publish or reproduce the published form of this document, or allow others to do so, for United States Government purposes. 


\title{
A precise in situ calibration of the $\mathrm{RHIC}$ H-Jet polarimeter
}

\author{
A.A. Poblaguev \\ March 5, 2014
}

\begin{abstract}
Two new methods of calibration of the hydrogen jet target polarimeter (H-Jet) at RHIC are discussed. First method is based on the measurement of low amplitude signal time of fast particles penetrating through detector. The second, geometry based, method employs correlation between $z$-coordinate of the recoil proton and its kinetic energy. Both methods can be used for in situ calibration of the H-Jet polarimeter. These two methods are compared with a traditional calibration of the H-Jet which uses $\alpha$-sources.
\end{abstract}

\section{Introduction.}

Proton beam polarization measurement is an important element of the Relativistic Heavy Ion Collider (RHIC) [1] polarized proton program. The polarized atomic hydrogen gas jet target (H-Jet) polarimeter [2] is employed to measure average polarization of both RHIC beams, "blue" and "yellow". Schematically, the H-Jet polarimeter consist of jet-target atomic beam and six 16-channel silicon detectors as shown on Fig. 1.

At the H-Jet location, blue and yellow RHIC beams are vertically separated, so polarization of both beams can be measured simultaneously. In each detector, 8 strips
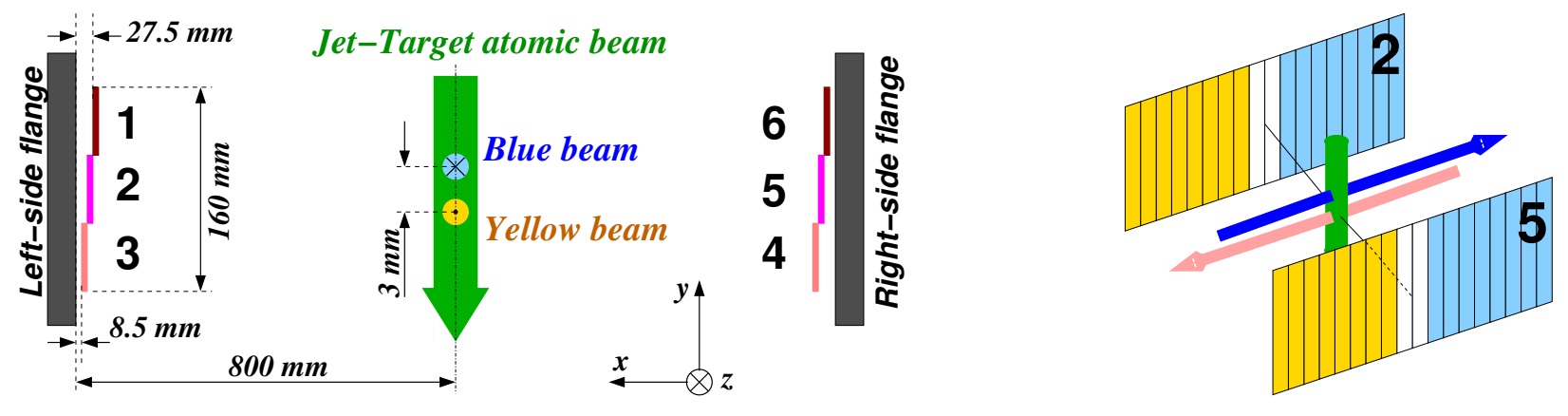

Figure 1: Schematic view of the hydrogen jet-target beam, the RHIC beams, and six silicon detectors (left). The H-Jet silicon detector division by strips (electronic channels), 8 strips per beam direction, is shown in the right picture. 

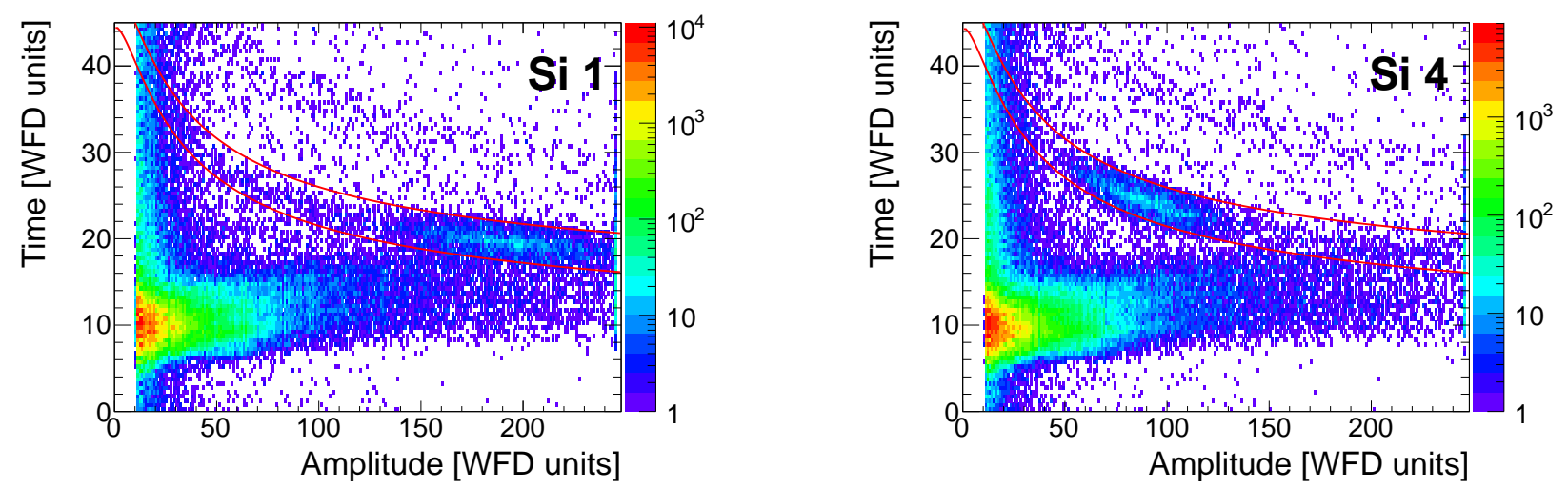

Figure 2: The dependence of measured time on signal amplitude for two silicon strips. The low energy recoil proton regions are isolated by red lines. The WFD units are $2.37 \mathrm{~ns}$ for time measurements and about $25 \mathrm{keV}$ of deposited energy for signal amplitude.

are designated to detect recoil protons produced by blue beam and eight strips to detect yellow beam recoil protons.

Determination of the beam polarization is based on the measurement of asymmetry of low energy (Coulomb Nuclear Interference region) recoil proton production in elastic $p^{\uparrow} p^{\uparrow}$ scattering. Since both, the proton beam and the jet target, polarizations are flipping, we can measure concurrently the asymmetry related to the beam and jet polarization:

$$
a_{\text {beam }}=A_{N} P_{\text {beam }}, \quad a_{\text {jet }}=A_{N} P_{\text {jet }}
$$

The average value of analyzing power $A_{N}$ is the same for the beam and jet asymmetry measurements because the event selection is identical for both studies. As result, the beam polarization may be related to the jet one

$$
P_{\text {beam }}=\frac{a_{\text {beam }}}{a_{\text {jet }}} P_{\text {jet }} .
$$

The H-Jet atomic hydrogen gas polarization $P_{\text {jet }} \approx 96 \%$ is monitoring by Breit-Rabi polarimeter. With this value known ${ }^{1}$, measurements of the recoil proton asymmetries $a_{\text {beam }}$ and $a_{\text {jet }}$ allow us to determine the absolute beam polarization $P_{\text {beam. }}$. For the recoil proton energy range $1-5 \mathrm{MeV}$, the average value of analyzing power is about $A_{N} \sim 0.04$. In the recent RHIC runs the proton beam polarization was measured to be about $55 \%$.

\section{Event selection for the polarization measure- ments}

The H-Jet Data Acquisition System (DAQ) records a 144 sample waveform with 2.37 ns sampling time for every triggered event [4]. The waveform analysis results in 2

\footnotetext{
${ }^{1}$ Taking into account an admixture of molecular hydrogen in the jet, the average polarization of the jet protons is about $P_{\text {jet }} \approx 92 \%[3]$.
} 

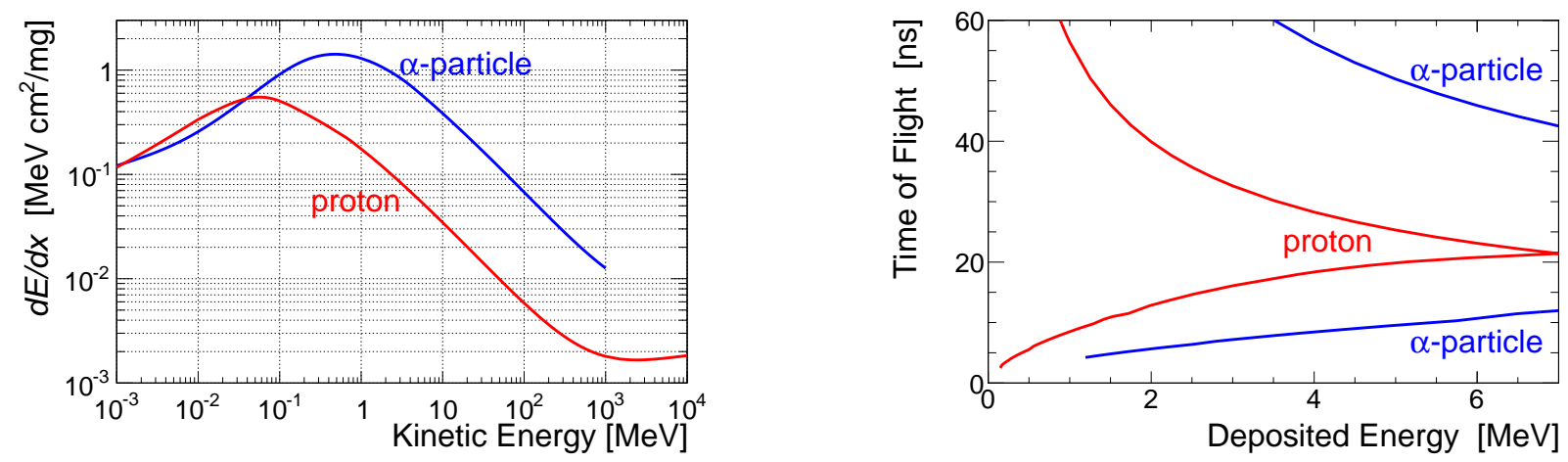

Figure 3: Silicon stopping power for proton and $\alpha$-particle [5] (left). The calculated dependence of the detected particle time of flight on energy deposited in the H-Jet detector (right).

measured parameters: amplitude $A$ and time $t$. A typical distributions of measured time versus amplitude in a silicon strip is shown in Fig. 2. The statistics is dominated by prompts, fast particles punched through silicon detector. Due to the dependence of stopping power on energy [5] (Fig. 3), the prompt signal time depends on the amplitude as

$$
t_{\text {prompt }} \approx t_{0}+c A^{0.6}
$$

The peaks at $A \sim 10$ and $t \sim 10$ in the Fig. 2 distributions are formed mostly by relativistic protons.

To isolate low energy recoil protons which are stopped in the detector, one may compare proton kinetic energy $T$ with its time of flight $t-t_{0}$ :

$$
\alpha A+E_{\mathrm{loss}}\left(\alpha A, x_{D L}\right)=T=\frac{M_{p} L^{2}}{2\left(t-t_{0}\right)^{2}}
$$

Here, $A$ and $t$ are measured amplitude and time of the signal, $M_{p}$ is proton mass, and $L \approx 80 \mathrm{~cm}$ is distance between interaction point and detector. To measure time of flight, the time offset $t_{0}$ has to be known. Energy deposited in the active part of detector $E_{\mathrm{dep}}=\alpha A$ is proportional to the gain $\alpha$. Part of the proton energy $E_{\text {loss }}$ is deposited in the detector entrance window (dead-layer) and does not contribute to the measured signal amplitude. Assuming that stopping power $d E / d x$ of silicon for protons [5] is known, the energy losses in the dead-layer may be calculated as a function of detected energy $\alpha A$ and dead-layer thickness $x_{D L}$.

Thus, to identify protons from the elastic $p p$ scattering one needs to know three calibration parameters $t_{0}, \alpha$, and $x_{D L}$ for every silicon strip. These parameters are supposed to be determined in a calibration.

\section{$3 \quad \alpha$-source calibration}

The H-Jet silicon detectors were usually calibrated using $\alpha$-sources [4]. In a designated calibration run, the silicon detectors are exposed by $\alpha$-particles emitted by ${ }^{148} \mathrm{Gd}$ 

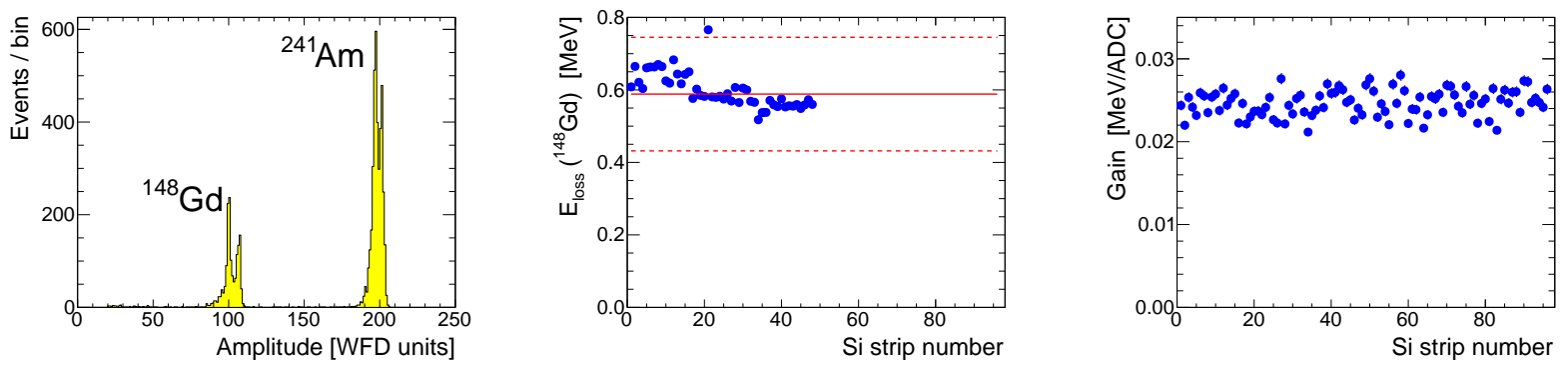

Figure 4: $\alpha$-source signals in a H-Jet silicon strip (left). Dead-layer thickness in units of energy lost by ${ }^{148} \mathrm{Gd}$ alpha (center). The solid red line is the average dead-layer which was used for gain calculations in all strips. Distribution of the gains in the silicon strips (right).
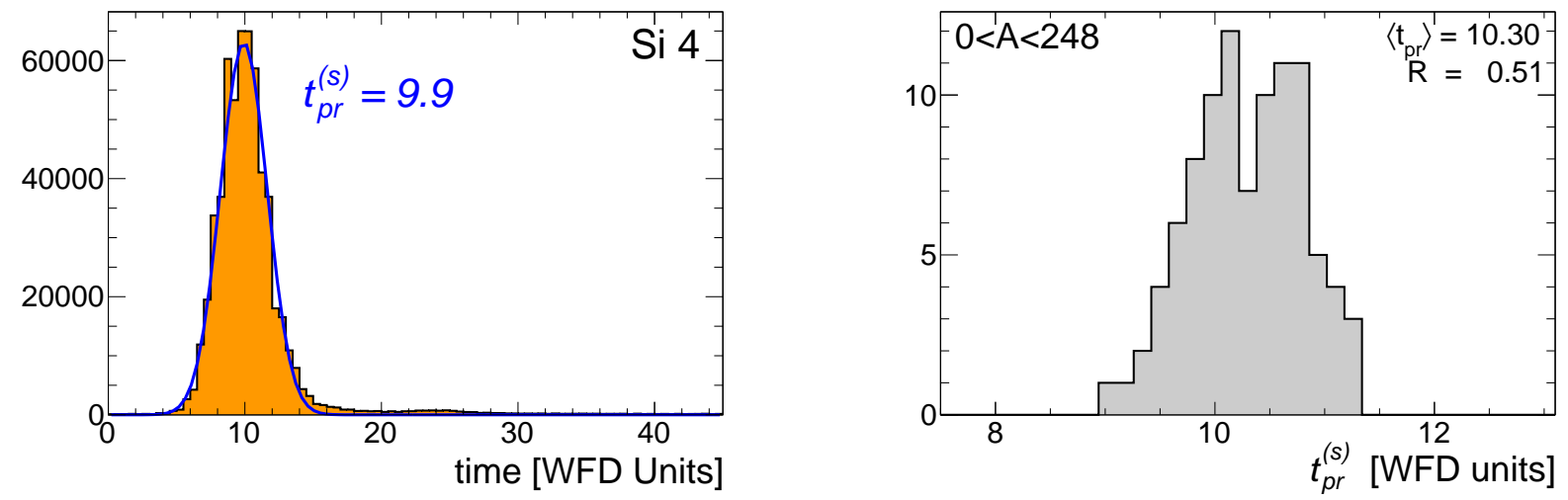

Figure 5: A distribution of measured time for all events in the silicon strip \#4 (left). A distribution of mean values of measured time for all 96 strips.(right).

(3.183 MeV) and ${ }^{241} \mathrm{Am}(5.486 \mathrm{MeV})$ sources. Two different energies of $\alpha$-particles allows one to determine two calibration parameters $\alpha$ and $x_{D L}$. An example of such a calibration is shown on Fig. 4 [6].

Only left-side detectors (1-3) are exposed by the Gd source. For these 48 channels, the average value of the dead-layer $\left\langle x_{D L}\right\rangle$ is calculated. For the 48 right side channels only Am signal is available. The gain for these channels is calculated assuming that dead-layer in each strip is equal to the $\left\langle x_{D L}\right\rangle$.

Time offset $t_{0}$ is not measured in the $\alpha$-calibration. Following Eq. (4), it may be calculated by analysis of the elastic $p p$ events.

\section{Determination of $t_{0}$ from the prompt events}

Since most events accepted by the DAQ are triggered by relativistic prompt particles (see Fig. 2), one may expect that mean measured time for these signals in a strip $c h$ is related to the time offset $t_{0}^{(s)}$ in this channel in a simple way:

$$
t_{\mathrm{pr}}^{(s)}=t_{0}^{(s)}+\Delta t
$$



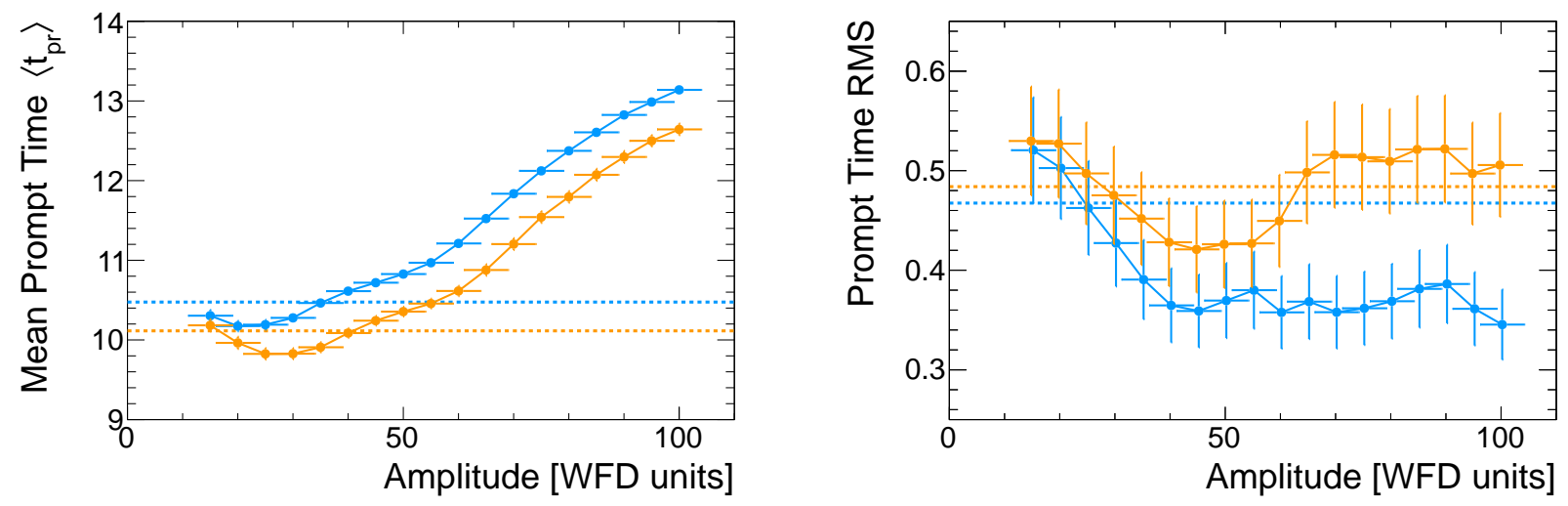

Figure 6: The dependence of mean prompt time $\left\langle t_{\mathrm{pr}}\right\rangle$ (left) and RMS (right) on signal amplitudes. The vertical error bars show RMS of the corresponding distributions. The dashed lines show values of $\left.t_{\mathrm{pr}}\right\rangle$ and $\mathcal{R}$ if amplitude cut is not applied. The dependencies are shown separately for the blue and yellow beams.

where $\Delta t=L / c \approx 2.67 \mathrm{~ns}$ is time of flight for a relativistic particle. The value of $\Delta t$ is supposed to be the same for all 96 silicon strips. A more detailed analysis shows that $\Delta t$ is larger than $L / c$ by $1-2 \mathrm{~ns}^{2}$ It means that $\Delta t$ should be experimentally measured by comparison of this method of determination of $t_{0}$ with other calibration methods.

A distribution of the measured prompt time in a H-Jet silicon strip is shown on Fig. 5. The value of RMS $\mathcal{R}$ on the right histogram, which is calculated as

$$
\mathcal{R}=\sqrt{\sum\left(t_{\mathrm{pr}}^{(s)}\right)^{2} / N_{s}-\left\langle t_{\mathrm{pr}}\right\rangle^{2}}, \quad\left\langle t_{\mathrm{pr}}\right\rangle=\sum t_{\mathrm{pr}}^{(s)} / N_{s}
$$

where $N_{s}=96$ is number of the silicon strips, may be approximated as the quadratic sum

$$
\mathcal{R}=\sigma_{\text {delay }} \oplus \sigma_{\mathrm{t} 0}
$$

of $\sigma_{\text {delay }}$, the variation of delays in electronic channels, and $\sigma_{\mathrm{t} 0}$, the variation of the evaluated values of $t_{0}$. Obviously, the value of the $\mathcal{R}$ sets an upper limit $\sigma_{\mathrm{t} 0} \leq \mathcal{R}=$ $1.2 \mathrm{~ns}$ for the accuracy of determination of the $t_{0}$ in such a calibration.

The described method of determination of the $t_{0}$ should be optimized by selecting the amplitude range for the calculation of $t_{\mathrm{pr}}$. The dependence of $\left\langle t_{\mathrm{pr}}\right\rangle$ and $\mathcal{R}$, separately for blue and yellow beam silicon strips, on the selected amplitude range is shown on Fig. 6. The minimum of the RMS, which corresponds the best accuracy of determination of $t_{0}$, is achieved if signal amplitudes are selected within the 40-50 WFD units interval.

\footnotetext{
${ }^{2}$ This difference can be explained by two main factors: (i) actual velocity of prompt particles is smaller then speed of light $c$ and (ii) due to charge collection effects in silicon detector the measured time for prompt particle is delayed by about 1 ns relative to the protons stopped in the detector [7]
} 


\section{Geometry based calibration}

Due to the geometrical orientation of the silicon strips, every strip detects recoil protons produced at a definitive angle $\theta_{R}$ only (see Fig. 1) This may be employed for a precise energy calibration of the detector.

\subsection{Proton energy spectrum in elastic $p p$ interaction}

For the elastic $p p$ scattering, $z$ (along the beam) coordinate of recoil proton in the detector is related to the proton kinetic energy $T$ as

$$
\frac{z}{L}=\tan \theta_{R}=\sqrt{\frac{T}{2 M_{p}} \frac{E_{\text {beam }}+M_{p}}{E_{\text {beam }}-M_{p}-T}}
$$

where $E_{\text {beam }}$ is beam proton energy ( $255 \mathrm{GeV}$ in the RHIC Run13). Neglecting the recoil proton kinetic energy $T$ compared to the beam energy $E_{\text {beam }}$, the $z$-coordinate may be expressed as

$$
z=\zeta \sqrt{T}
$$

with a known factor $\zeta$.

The distribution of detected events per momentum transfer invariant $t=-2 M_{p} T$ is proportional to

$$
\frac{d N}{d t} \propto \frac{d \sigma_{p p}}{d t} \int d z_{j} n\left(z_{j}\right) \mathcal{A}\left(z_{j}, z_{s}, T\right)
$$

where $d \sigma_{p p} / d t$ differential cross section of elastic $p p$ scattering, $z_{j}$ is the scattering coordinate, $n\left(z_{j}\right)$ is $z$-coordinate distribution of proton density in the jet, and $\mathcal{A}\left(z_{j}, z_{s}, T\right)$ is acceptance of detecting of recoil proton with kinetic energy $T$ in a strip with $z$ coordinate $z_{s}$. Proton's density in the jet may be parametrized by Gaussian distribution

$$
n\left(z_{j}\right) \propto \exp \frac{-0.5 z_{j}^{2}}{(2.7 \mathrm{~mm})^{2}}
$$

For a very narrow silicon strip the acceptance is approximated by delta function:

$$
A\left(z_{j}, z_{s}, T\right) \propto \delta\left(z_{s}-z_{j}-\zeta \sqrt{T}\right)
$$

and energy distribution of detected events in such a strip has a simple dependence on $\sqrt{T}$

$$
\frac{d N}{d t} \propto \frac{d \sigma_{p p}}{d t} \times n\left(z_{s}-\zeta \sqrt{T}\right)
$$

Thus, for the elastic $p p$ scattering and for a very narrow strip, the function

$$
\eta(\sqrt{A})=\left(\frac{d \sigma_{p p}}{d t}\right)^{-1} \frac{d N}{d A},
$$

gives the image of the jet proton concentration along $z$-axis. The signal amplitude $A$ is assumed to be proportional to the proton kinetic energy $T$. The value of amplitude, $A_{s}$, at which the function $\eta(\sqrt{A})$ has maximum, may be associated with a well defined strip energy $T_{s}=z_{s}^{2} / \zeta^{2}$.

The conclusion about the function $\eta(\sqrt{A})$ was done in a simplified picture of detection of recoil protons. However, it should remain a good approximation in analysis of real data. 
- Silicon strip width. A finite width $d=4.44 \mathrm{~mm}$ of the strip may be accounted by smearing of the jet density:

$$
n\left(z_{j}\right) \Rightarrow n_{\mathrm{eff}}\left(z_{j}\right) \propto \exp \frac{-0.5 z_{j}^{2}}{(3.0 \mathrm{~mm})^{2}}
$$

In such an approximation, the result of measurement of the $A_{s}$ is not affected by the strip width.

- Background. The background protons in the H-Jet measurements are dominated by beam scattering on the beam line gas. Such a scattering is expecting to produce the same background in all strips and, thus, the background may be experimentall evaluated by comparing measured function $\eta(\sqrt{A})$ in different strips. It was also found that description of the background by a flat distribution in $\sqrt{A}$ is a sufficiently good approximation to begin with.

- Non-linearity $A(T)$ of the measured amplitude dependence on proton energy. In fact, signal amplitude is not proportional to the proton kinetic energy. For example, almost $10 \%$ of $1 \mathrm{MeV}$ proton energy is lost in the dead-layer. The problem may be solved by conversion of the measured amplitude $A$ to the kinetic energy using pre-calibration and iterative repeating of the calibration procedure. However, as it will be shown in section 7 , ignoring the problem, i.e. continuing to use $A$ instead of $T$ will result only in negligible systematic errors in the calibration.

- Holding magnetic field. The jet polarization is controlled by holding magnetic fields. In order to minimize the effect of this field on the recoil protons, the Nested Opposing Helmholtz-Type Coils are used in the H-Jet polarimeter. The fields are adjusted to keep the total vertical field integral along the proton path close to zero. Nonetheless, inaccuracy of the field adjustment results in bending of proton tracks which essentially violate the Eq. (8). The corrections to this equation will be discussed in section 5.3.
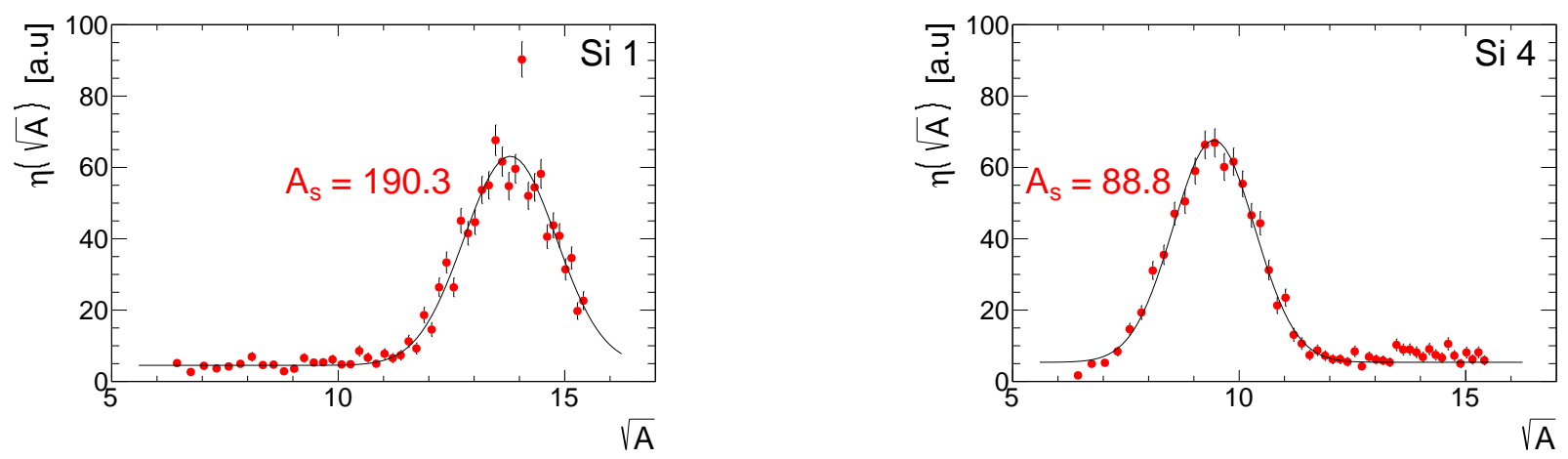

Figure 7: The measured function $\eta(\sqrt{A})$ for two silicon strips in one detector. The distance between strip centers is $13.3 \mathrm{~mm}$. 


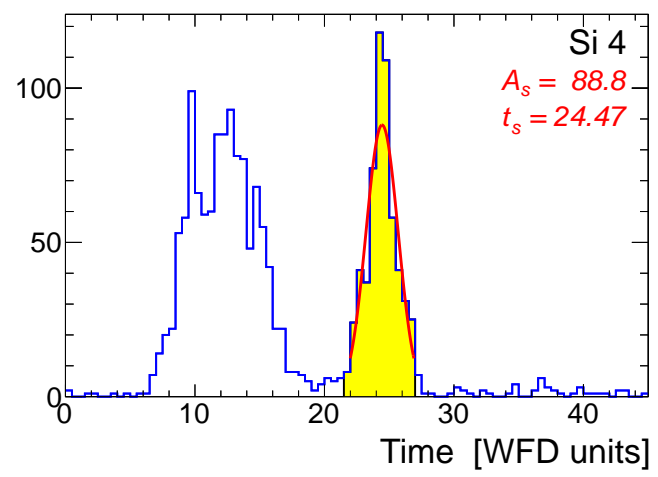

Figure 8: Measurement of the signal time at amplitude $A_{s}$ corresponding to the maximum of the function $\eta(\sqrt{A})$. The width of the measured time distribution is dominated by the RHIC bunch length.

\subsection{The calibration schema}

The experimentally measured function $\eta(\sqrt{A})$ for two silicon strips (with different coordinates $\left.z_{s}\right)$ is shown in Fig. 7 . To determine the $A_{s}$, one can fit the $\eta(\sqrt{A})$ with a function

$$
p+a \exp \left[-\frac{\left(\sqrt{A_{s}}-\sqrt{A}\right)^{2}}{2 \sigma^{2}}\right]
$$

with 4 parameters: $p, a, \sigma$, and $A_{s}$. The value of $A_{s}$ found in the fit may be associated with the strip energy $T_{s}$

$$
E\left(A_{s}\right)=T_{s}=2 M_{p}\left(\frac{z_{s}}{L}\right)^{2} \frac{E_{\text {beam }}-M_{p}}{E_{\text {beam }}+M_{p}}
$$

where $z_{s}$ is $z$-coordinate of the strip relative to the center of jet. The value of $t_{0}$ can be calculated as a difference between measured signal time $\left\langle t_{s}\right\rangle$ for the amplitude $A_{s}$ (see Fig. 8) and time of flight for proton with kinetic energy $T_{s}$ :

$$
t_{0}^{(g)}=\left\langle t_{s}\right\rangle-L \sqrt{\frac{M_{p}}{2 T_{s}}}
$$

The accuracy of the energy calibration $\sigma_{T} / T$ could be related to the accuracy $\sigma_{t 0}$ of determination of the $t_{0}$. As it follows from Eq. (4)

$$
\frac{\delta T}{T}=-\frac{2 \delta t}{t} \approx-\frac{2 \sqrt{T / \mathrm{MeV}}}{57.7 \mathrm{~ns}} \delta t \Rightarrow \sigma_{T} / T=\frac{\sigma_{t 0} \sqrt{T / \mathrm{MeV}}}{28.9 \mathrm{~ns}}
$$

Here, $\sigma_{t 0}$ is accuracy of determination (calibration) of the $t_{0}$. To measure proton energy with a percent accuracy $0.7 \sqrt{T / \mathrm{MeV}} \%$, we have to know the $t_{0}$ with accuracy better than $\sigma_{t 0} \leq 0.2 \mathrm{~ns}$.

The value of $\sigma_{t 0}$ can be experimentally estimated by comparison of values of the prompt time of flight $\Delta t=\left\langle t_{\mathrm{pr}}^{(s)}\right\rangle-t_{0}^{(g)}$ (5) for all silicon strips. Fig. 9 shows a very 

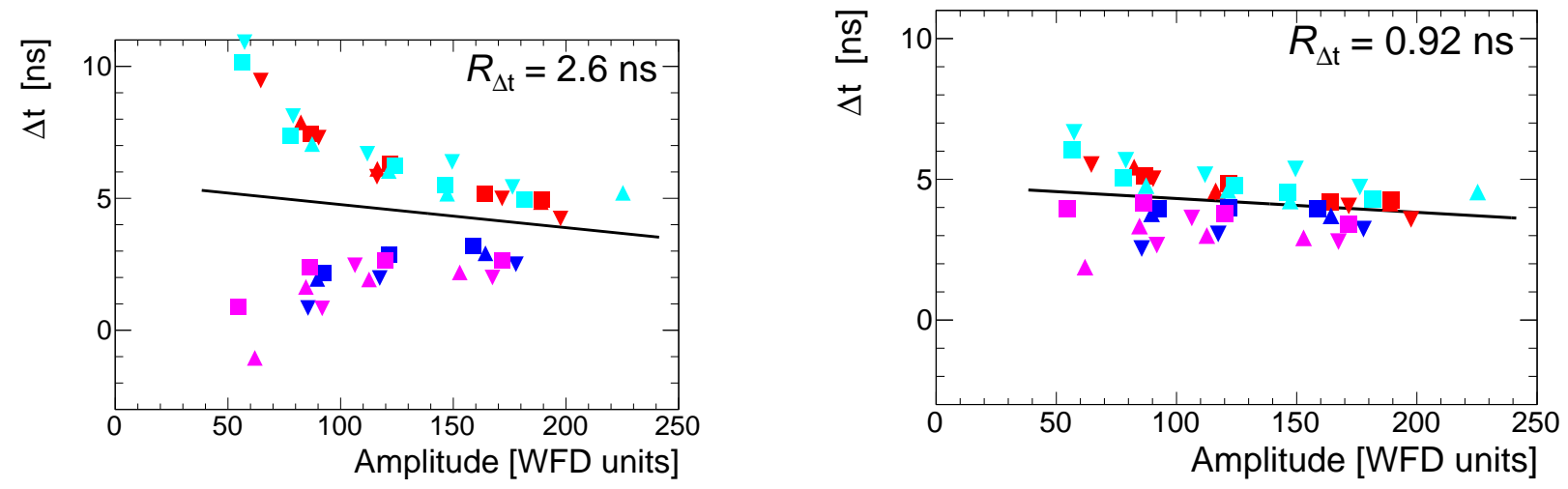

Figure 9: The dependence of the difference between prompt time $\left\langle t_{\mathrm{pr}}^{(s)}\right\rangle$ and $t_{0}^{(g)}$ on the signal amplitude $A_{s} . \quad R_{\Delta t}$ is RMS of the time difference $\Delta t$ distribution. Silicon strips belonging to different detectors are separated by marker color and shape. No geometry alignment corrections are applied on the left plot. The holding magnetic field corrections $(b=9 \mathrm{MeV} \cdot \mathrm{cm})$ only are applied on the right plot.

poor consistency between these two methods of calibration. With such a discrepancy in $\Delta t$, we can measure the recoil proton energy with an accuracy no better than $10 \%$. However, it is clearly seen that values of $\Delta t$ are strongly correlated with left/right location of the detector and the beam direction. Such a discrepancy may be eliminated by proper alignment of the detectors.

\subsection{Geometrical alignment}

The geometry based calibration values of $t_{0}^{(g)}$ used in Fig. 9 were calculated assuming ideal geometrical alignment of detectors, target jet, and RHIC beams, i.e. it was assumed that $z$ coordinates of centers of all detectors and the jet are equal to 0 and yellow and blue beams are collinear the $z$-axis. In general, this assumption is not necessary valid. If we use the centers of detectors 2 and 5 to define the axis $x$ (see Fig. 10), then general geometrical configuration of the H-Jet may be described by $z$-coordinates, $z_{1}, z_{3}, z_{4}, z_{6}$, of remaining 4 detectors, the jet center coordinate $z_{\text {jet }}$ and blue and yellow beam direction angles $\theta_{B}$ and $\theta_{Y}$. Instead of the $\theta_{B}$ and $\theta_{Y}$ we can use the average beam angle $\theta=\left(\theta_{Y}+\theta_{B}\right) / 2$ and the angle between blue and yellow beams $\delta \theta=\theta_{B}-\theta_{Y}$.

In addition to the geometrical alignment offset, the relationship (8) between $z$ coordinate of recoil proton in the silicon detector and its energy is modified by bending of proton track in the holding magnetic field (Fig. 11):

$$
\frac{z}{L}=\sqrt{\frac{T}{2 M_{p}} \frac{E_{\text {beam }}+M_{p}}{E_{\text {beam }}-M_{p}}} \pm \frac{b}{L \sqrt{2 M_{p} T}}
$$

Sign \pm depends on left/right position of detector relative to the beam direction. The 


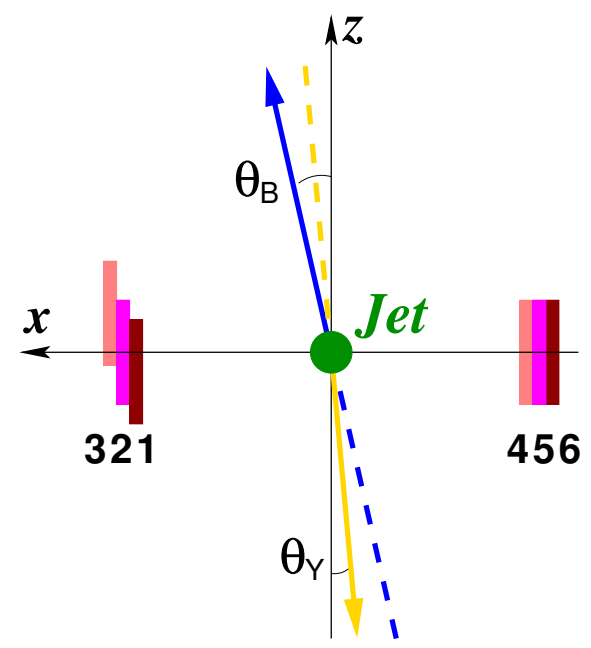

Figure 10: A schematic plan view of possible misalignment of the H-Jet detectors, the RHIC beams, and the Jet.

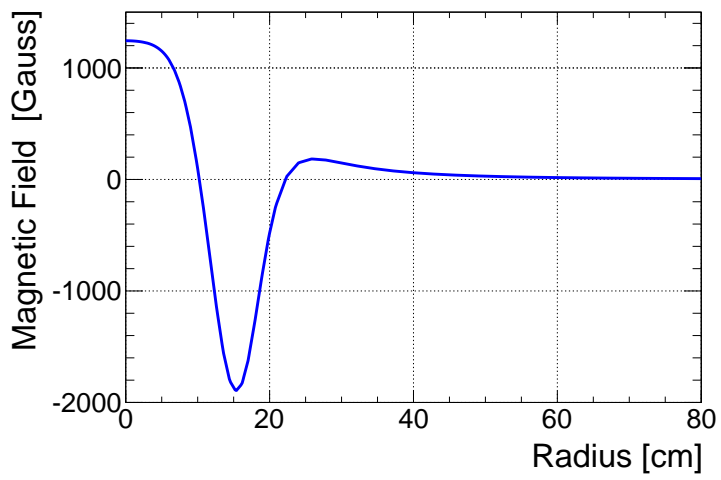

Figure 11: The holding magnetic field as a function of distance from the Jet center.

parameter $b$ could be calculated if holding magnetic field is known

$$
b=\left(\frac{q L}{c}\right) \times \int_{0}^{L} H(r)\left(1-\frac{r}{L}\right) d r
$$

where $q L / c=2.4 \times 10^{-2} \mathrm{MeV} / \mathrm{G}$. For the field in Fig. $11, b=27 \mathrm{MeV} \cdot \mathrm{cm}$. Since a small value of $b$ is calculated as a difference of large values of positive and negative magnetic fields, such a calculation of the parameter $b$ is not reliable. For on-line analysis of the H-Jet data, the value of $b=9 \mathrm{MeV} \cdot \mathrm{cm}$ was used in recent RHIC runs.

The geometry based calibration allows us to determine all geometry alignment parameters $z_{1}, z_{3}, z_{4}, z_{6}, z_{\text {jet }}, \theta, \delta \theta$, and $b$. For that we should minimize, by varying these parameters, a variation in the values of $\Delta t$ measured in all silicon strips. It should be noted that there is a strong correlation between $\theta$ and $b$. Actually, holding field effect may be considered as the beam angle $\theta$ depending on the recoil proton energy as $\theta \sim 1 / \sqrt{T}$ 


\begin{tabular}{lc|r|r} 
& & Fill 17247 & Fill 17600 \\
\hline \hline$z_{1}$ & $(\mathrm{~mm})$ & $0.17 \pm 0.24$ & $0.33 \pm 0.16$ \\
$z_{3}$ & $(\mathrm{~mm})$ & $-0.09 \pm 0.27$ & $0.07 \pm 0.17$ \\
$z_{4}$ & $(\mathrm{~mm})$ & $-0.38 \pm 0.22$ & $-0.36 \pm 0.14$ \\
$z_{6}$ & $(\mathrm{~mm})$ & $-0.64 \pm 0.22$ & $-0.54 \pm 0.24$ \\
$z_{\text {jet }}$ & $(\mathrm{mm})$ & $0.13 \pm 0.11$ & $-0.05 \pm 0.07$ \\
$\theta$ & $(\mathrm{mrad})$ & $0.35 \pm 0.60$ & $-0.10 \pm 0.39$ \\
$\delta \theta$ & $(\mathrm{mrad})$ & $0.07 \pm 0.18$ & $-0.04 \pm 0.12$ \\
$b$ & $(\mathrm{MeV} \cdot \mathrm{cm})$ & $9.7 \pm 2.8$ & $12.4 \pm 1.9$
\end{tabular}

Table 1: The alignment parameters determined in two RHIC fills 17247 (March 25, 2013) and 17600 (June 8, 2013).

Determination of geometry alignment parameters for two RHIC fills are summarized in Table 1. One may note a good consistency between results of these two alignments. Only 4 alignment parameters, parameters $z_{1}, z_{4}, z_{6}$, and $b$, indicated nonzero values.

\section{Comparison of the calibrations}
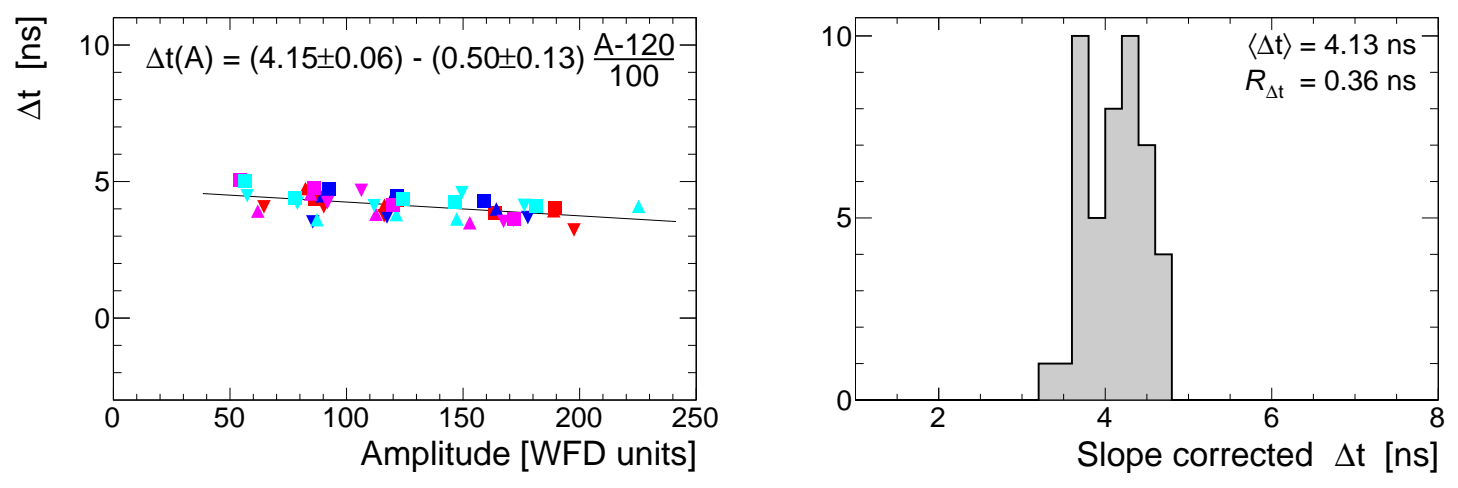

Figure 12: The $\Delta t$ distribution after geometrical alignment.

Taking into account the alignment corrections, the comparison of prompt time and $t_{0}$ determined in geometry based calibration (Fig. 12) may be digitized as:

$$
\sigma_{t 0}^{(g)} \oplus \sigma_{t 0}^{(p)}=0.36 \mathrm{~ns}
$$

Here $\sigma_{t 0}^{(g)}$ and $\sigma_{t 0}^{(p)}$ are accuracies of determination of $t_{0}$ in geometry and prompt calibrations, respectively, and $\oplus$ means quadratic summation.

In a similar way we may compare these calibration with the $\alpha$-source calibration. For that we can calculate $t_{0}^{(\alpha)}$ by substituting in Eq. (19) the $T_{s}$ with $E_{\alpha}\left(A_{s}\right)$ where 
$E_{\alpha}$ is energy calculated from the measured amplitude using alpha calibration.

$$
\begin{aligned}
& \sigma_{t 0}^{(g)} \oplus \sigma_{t 0}^{(\alpha)}=0.29 \mathrm{~ns} \\
& \sigma_{t 0}^{(p)} \oplus \sigma_{t 0}^{(\alpha)}=0.49 \mathrm{~ns}
\end{aligned}
$$

From such a comparison we should conclude that geometry based calibration provides best accuracy of determination of $t_{0}$ with a conservative estimate of

$$
\sigma_{t 0}^{(g)} \lesssim 0.20 \mathrm{~ns}
$$

A combined accuracy of determination of $t_{0}$ using all three calibration methods may be estimated as

$$
\sigma_{t 0} \approx 0.10 \div 0.15 \mathrm{~ns}
$$

which corresponds to the accuracy of energy calibration better than $1 \%$ for $1 \div 5 \mathrm{MeV}$ protons.
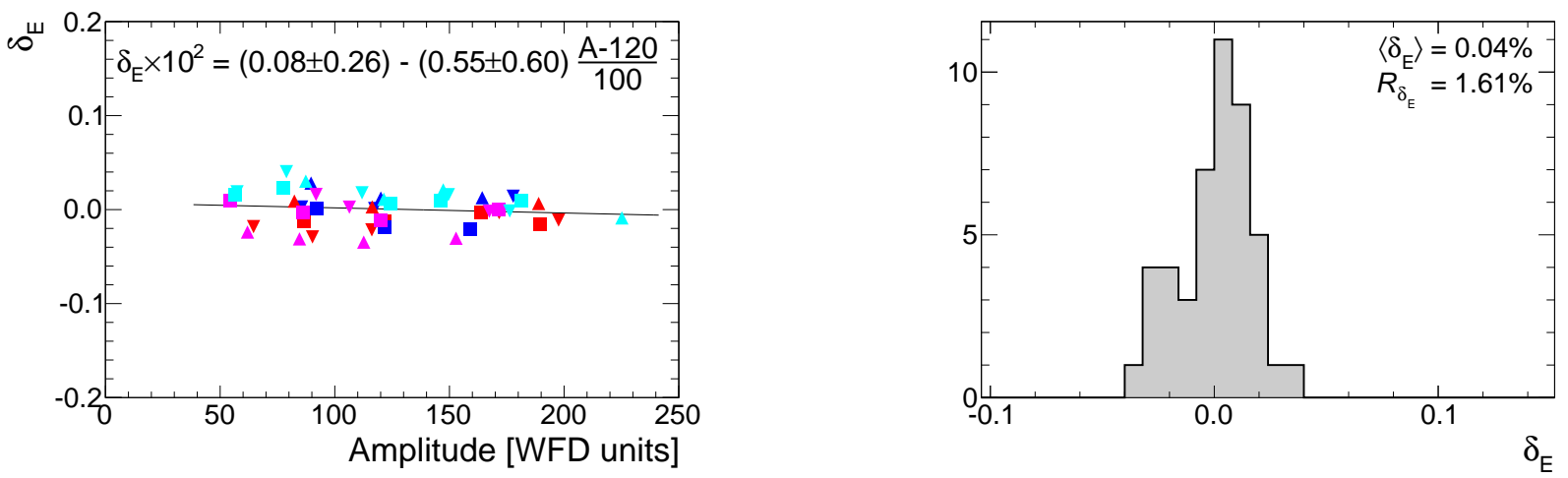

Figure 13: Comparison of the geometry based and $\alpha$-calibrations.

The $\alpha$-source and geometry based calibrations may be compared directly without calculations of $t_{0}$. For a particular silicon strip, a discrepancy between 2 calibrations may be parametrized as

$$
\delta_{E}=2 \frac{T_{s}-E_{\alpha}\left(A_{s}\right)}{T_{s}+E_{\alpha}\left(A_{s}\right)}
$$

The distribution of this discrepancy is shown on Fig. 13. It may be interpreted as

$$
\begin{aligned}
\left\langle\delta_{E}\right\rangle & =0.0 \pm 0.3 \% \\
\sqrt{\left\langle\delta_{E}^{2}\right\rangle} & =\left\langle\frac{\sigma_{E}}{E}\right\rangle_{\text {geom }} \oplus\left\langle\frac{\sigma_{E}}{E}\right\rangle_{\alpha}=1.6 \pm 0.3 \%
\end{aligned}
$$

It has to be mentioned that no dependence of the $\delta$ on signal amplitude was observed.

A small correlation between the value of $\delta_{E}$ and the silicon detector location is being observed in Fig. 13. A possible source of the observed discrepancy is the dead-layer dependence on detector number (which were not accounted in the $\alpha$-source calibration). A further study of this systematics promises an improvement of the consistency between the geometry and $\alpha$-source based calibrations. 


\section{$7 \quad t_{0}$ dependence on signal amplitude.}

A straightforward interpretation of the linear dependence of $\Delta t$ on signal amplitude $A$ (see Fig. 12) is a dependence of the $t_{0}^{(g)}$ on $A$

$$
t_{0}=\text { const }+0.005 A \mathrm{~ns}
$$

According to the Eq. (18) such a dependence may be caused by $A$-correlated systematic errors either in measurement of the $\left\langle t_{s}\right\rangle$ or in determination of the $T_{s}$.

Simulation of the charge collection in silicon detector [7] indicate some dependence of signal waveform and, thus, measured time on signal amplitude. However, the dependence found in the calculation can not explain the Eq. (30).

Potentially, a $10 \%$ systematic error in determination of the $T_{s}$ (for example, due to the wrong values of the $L$ or silicon strip width used in the data analysis) can emulate the observed dependence of the $t_{0}$ on measured amplitude $A$. However, a good agreement between the $T_{s}$ and $E_{\alpha}\left(T_{s}\right)$, i.e. between $\alpha$-source and geometry based calibrations, rules out such an explanation of the Eq. (30).

The geometry based calibration, as it was described above, assumes the linear dependence of measured amplitude on proton kinetic energy. For completeness we should estimate possible non-linear effects. For that, we may consider a scaled kinetic energy $\tau$ approximately equal to the measured amplitude $A$.

$$
\tau=A+a(A)
$$

Here, $a(A)$ is the correction to the amplitude due to the non-linearity. Actually the $\tau$ should be used in Eqs. (14) and (16). If the measured signal amplitude $A$ is used instead, the jet $z$-coordinate profile will be, in first order approximation, modified as

$$
\exp \left(-\frac{\left(\sqrt{\tau}-\sqrt{\tau_{s}}\right)^{2}}{2 \sigma^{2}}\right) d \sqrt{\tau} \Rightarrow \exp \left(-\frac{\left(\sqrt{A}-\sqrt{A_{s}}-\delta A / 2 \sqrt{A}\right)^{2}}{2 \sigma^{2}}\right) d \sqrt{A}
$$

Here, $A_{s}$ is an amplitude corresponding to the strip energy $T_{s}, \tau_{s}=A_{s}=a\left(A_{s}\right), \sigma \approx 1$ (see Fig.(7), and

$$
\delta A=2 \sigma^{2}\left(\frac{a\left(A_{s}\right)}{A_{s}}-a^{\prime}\left(A_{s}\right)\right)
$$

is actually an error in determination of $A_{s}$ due to the non-linearity. As result, the systematic error in determination of $\Delta t$ is acquired:

$$
\Delta t_{\text {meas }}=\Delta t+\frac{\delta A}{2 A_{s}} \sqrt{A_{n}} t_{n}
$$

where $A_{n}$ is arbitrarily chosen normalization amplitude and $t_{n}$ is time of flight corresponding to this amplitude. If $5 \mathrm{MeV}$ proton signals are selected for such a normalization then

$$
A_{n} \approx 200, \quad t_{n}=25.8 \mathrm{~ns} .
$$

If we assume a power dependence of the nonlinear correction on signal amplitude

$$
a(A)=a_{n}\left(\frac{A}{A_{n}}\right)^{p}
$$


then

$$
\Delta t_{\text {meas }}=\Delta t+t_{n} \frac{\sigma^{2}}{A_{n}} \frac{a_{n}(1-p)}{A_{n}}\left(\frac{A}{A_{n}}\right)^{p-5 / 2} .
$$

Two obvious nonlinear effects should be considered

- Energy loss in dead-layer. Since the power stop in silicon for $1-5 \mathrm{MeV}$ protons may be approximated as $d E / d x=177 /(T / \mathrm{MeV})^{0.68} \mathrm{MeV} \mathrm{cm}^{2} / \mathrm{g}$ we find $p \approx$ $-0.68, a_{n} / A_{n}=(d E / d x) \times x_{D L}$, and

$$
\Delta t_{D L}=\Delta t+\frac{0.29 \mathrm{~ns}}{(T / \mathrm{MeV})^{3.2}}
$$

Such a correction is inessential for the distribution on Fig. 12.

- Signal saturation. Even for a hypothetic $10 \%$ saturation of the $T=5 \mathrm{MeV}$ proton signal $A \propto T(1-0.02 T)$ (i.e. if $p=2$ and $a_{n} / A_{n}=0.1$ ), we find

$$
\Delta t_{\mathrm{sat}}=\Delta t+\frac{0.03 \mathrm{~ns}}{\sqrt{T / \mathrm{MeV}}}
$$

which is negligible.

At moment we have no good understanding of the $\Delta t$ dependence on amplitude. Taking into account the following observations: (i) there is a good agreement between measured values of $T_{s}$ and $E_{\alpha}\left(A_{s}\right)$ and (ii) testing of Eq. (4) in a single strip indicate a similar dependence of $\Delta t$ on signal amplitude, we should relate the observed effect to a dependence of the measured signal time on amplitude or, in other words, to the dependence of $t_{0}$ on $A$. In such an interpretation of Fig. 12, the time offset $t_{0}$ in every channel is related to the prompt mean time as

$$
t_{0}^{(c h}=\left\langle t_{\mathrm{pr}}^{(s)}\right\rangle-4.75+0.005 A \mathrm{~ns}
$$

\section{Summary}

With two methods described in this note we have three independent calibration methods of the H-Jet detectors. Neither method provides a full calibration which includes determination of the ADC gain $\alpha$, dead-layer thickness $x_{D L}$, and time offset $t_{0}$. However, each calibration may be completed by testing elastic $p p$ signals with Eq. (4). It was found a good agreement between all 3 methods.

The geometry based calibration gives best results, but it can be used only for about half of all silicon strips. A combined use of geometry and prompt time base calibrations allows us to calibrate all silicon strips in situ. Contrary to the $\alpha$-calibration, these two methods does not require a standalone measurement.

After calibration, the recoil proton kinetic energy could be measured with accuracy better than $\sigma_{T} / T \sim 1 \%$ in the energy range of $1-5 \mathrm{MeV}$.

\section{References}

[1] I. Alekseev et al. NIM A499 (2003) 392; 
[2] H. Okada et al., Phys.Lett. B638 (2006) 450.

I. G. Alekseev et al., Phys. Rev. D79:094014,2009

[3] Y. I. Makdisi et. al., AIP Conf. Proc. 915, 975 (2007).

[4] Hiromi Okada, Measurement of the Analyzing Power $A_{N}$ in pp Elastic Scattering in the CNI Region with a Polarized Atomic Hydrogen Gas Jet Target, $\mathrm{PhD}$ Thesis. Kyoto University 2006

http://www.bnl.gov/userscenter/Thesis/2007/Okada_Thesis.pdf

[5] http://physics.nist.gov/PhysRefData/Star/Text/PSTAR.html

ICRU (1993). International Commission on Radiation Units and Measurements.

ICRU Report 49, Stopping Powers and Ranges for Protons and Alpha Particles.

[6] K. O. Eyser, private communication.

[7] A. Poblaguev, Waveform dependence on signal amplitude in the RHIC H-Jet polarimeter, (unpublished). 